\title{
The Ability of $\alpha$-Conidendrin-decomposing Agrobacterium Strains to Utilize Other Lignans and Lignin-related Compounds
}

\author{
BY VERONICA SUNDMAN \\ Department of Microbiology, University of Helsinki, Pihlajamäki, Finland
}

(Received 11 September 1963)

\section{SUMMARY}

By the application of respirometric techniques, it was found that the $\alpha$-conidendrin (I)-decomposing agrobacteria included strains which were adaptively or constitutively lignanolytic. The lignans isotaxiresinol (V), iso-olivil (VI), and olivil (VII) were rapidly oxidized by all the bacteria examined. The adaptive organisms showed a time-lag before oxidation of the lignans if they had been grown on a conidendrin-free medium. The lag required for oxygen uptake with $\alpha$-conidendrin was shorter than that observed for the other lignans. Matairesinol (IV), which differs structurally from $\alpha$-conidendrin through the lack of the 'isolignan' bond was not attacked by any of the agrobacteria examined. Olivil and iso-olivil were oxidized identically, giving results which indicate that the organisms bring about the isomerization olivil $\rightarrow$ iso-olivil. $\alpha$-Conidendrol (II) was indicated by the simultaneous adaptation to be an intermediate in $\alpha$ conidendrin decomposition. All the bacteria studied possess a constitutive ability to oxidize aromatic aldehydes (vanillin, isovanillin, veratrumaldehyde, syringaldehyde). By calculation from the total oxygen uptake, and by paper chromatography, it was found that these oxidations gave the corresponding acids. Simultaneously with oxidation to vanillic acid, a small amount of vanillin was reduced to vanillyl alcohol. Coniferyl alcohol was oxidized to ferulic acid.

\section{INTRODUCTION}

$\alpha$-Conidendrin (Fig. 1, I) like other lignans is synthesized by a variety of plants. It has been assumed to originate in the same simple $\mathrm{C}_{6} \mathrm{C}_{3}$-precursors as lignin (Erdtman, 1955). Spruce wood contains $0.048 \%(\mathrm{w} / \mathrm{w}) \alpha$-conidendrin according to Freudenberg \& Knof (1957). During the sulphite cooking process $\alpha$-conidendrin is enriched in the sulphite waste liquors of various sap woods, and can conveniently be isolated from these sources. Consequently the lignan is readily available, and has found use as a dimeric model compound for the guaiacylpropan unit of lignin, in work on biological lignin degradation. There have been numerous demonstrations of the presence of $\alpha$-conidendrin-decomposing bacteria in the most diverse surroundings rich in wood residue (Konetzka, Pelczar \& Gottlieb, 1952; Tabak, Chambers \& Kabler, 1959; Sundman, 1962). The general occurrence of $\alpha$-conidendrin-decomposing bacteria of different genera in material rich in plant residue might indicate that in nature these organisms also attack lignin-related structures other than $\alpha$-conidendrin, which as mentioned above represents a diminutive part only of the wooden plant. 
Previous workers on the subject have attempted to show, mostly with negative results, that $\alpha$-conidendrin-degrading bacteria utilize other lignin-related material. Pratt, Konetzka, Pelczar \& Martin (1953) studied a flavobacterium, isolated by Konetzka et al. (1952) which caused complete degradation of $\alpha$-conidendrin (supplied as $0.5 \%, \mathrm{w} / \mathrm{v}$ ), in 14 days, but it did not use a variety of lignin preparations and lignin-related monomers as the source of carbon + energy; of a number of lignin-related substances tested, the only compound which permitted luxuriant growth was vanillic acid. Tabak et al. (1959), who studied $\alpha$-conidendrin degradation by mixed and pure cultures of various Pseudomonas, Flavobacterium, and Achromobacter species, tested commerical lignin residues as sources of energy for the cultures, but found no evidence for utilization of the lignin preparations.

A description has been given of the $\alpha$-conidendrin-decomposing Agrobacterium strains used in the present work (Sundman, 1962, 1964). These exhibit many metabolic differences from what is known from previous work on $\alpha$-conidendrindegrading bacteria. In conformity with its suitability as an energy source for the Flavobacterium of Konetzka, vanillic acid was found to occupy a leading position in the reactions which lead to ring cleavage of the conidendrin molecule brought about by some Flavobacterium and Pseudomonas species (Konetzka, Woodings \& Stove, 1957; Tabak et al. 1959). In contrast, in the present work, the agrobacteria examined did not grow with vanillic acid as the sole carbon + energy source, and it was found that they did not produce vanillic acid from $\alpha$-conidendrin but instead, considerable quantities of isovanillic acid (Sundman, 1962). The efficiency to decompose $\alpha$-conidendrin was rapidly lost by the cultures of Tabak et al. (1959) when they were grown, for example, with yeast extract as the carbon + energy source, whereas some of the present organisms have been kept for years on yeast extract without losing the ability to decompose $\alpha$-conidendrin. The Flavobacterium of Konetzka and the cultures of Tabak et al. (1959), synthesized polysaccharides during growth on $\alpha$-conidendrin but were unable to use simple carbohydrates as a carbon + energy source (Pratt et al. 1953). The agrobacteria used in the present work have not been found to produce any polysaccharide concurrently with $\alpha$-conidendrin degradation, and they can grow on a variety of simple carbohydrates (Sundman, 1964).

Accordingly, there is justification for assuming that there exist different modes of action among the lignanolytic soil bacteria, and different types of bacterial attack on the dimeric guaiacylpropane structure as it occurs in the lignans. It was felt that a more detailed study of the agrobacteria-like $\alpha$-conidendrin-decomposing bacteria, as regards ability to oxidize other lignans and lignin 'model compounds', might provide data about the part they may play in oxidative metabolism which leads to release of the relatively resistant lignin-carbon back to atmospheric carbon dioxide. The present paper describes the examination of the oxidative activity of washed organisms on a number of lignans and monomeric guaiacyl- and syringylderivatives.

\section{METHODS}

Organisms. The Agrobacterium strains ks1741, Ms144412 and o811 previously described (Sundman, 1964) were used. The technique for the production of suspensions of washed organisms was also outlined in that paper. The rate of oxygen uptake/unit quantity of organism varied from batch to batch, and a decrease in 
oxygen-uptake activity with the lignans as substrate was observed during storage in the refrigerator, although the endogenous respiration remained constant during storage up to 10 days. Thus, the results obtained in different experiments are not directly comparable, even when the quantity of organism added to the Warburg flasks was identical. The period of storage in the refrigerator before measurement is stated where the results obtained with organisms of different age are presented in the same figure.

Manometry. Unless otherwise noted, the respirometric experiments were made in $\mathrm{M} / 45$-phosphate buffer at $\mathrm{pH} \mathbf{5 \cdot 9}$. When examining the effect of $\mathrm{pH}$ value, the final phosphate-concentration used was $\mathrm{m} / 30$. For determinations of the total oxygen uptake suspensions of organism were added from the side arm, the substrate being in the main compartment. Manometer readings were made at 15 -min. intervals; the results are presented with intervals of 30 or $60 \mathrm{~min}$., and give means of duplicate manometer readings. For further details see Sundman (1964).

Evaluation of growth on defined carbon source. As described by Sundman (1964), washed organisms were inoculated into semi-solid mineral medium containing $10^{-3}$ or $10^{-4} \mathrm{~mole} / \mathrm{ml}$. of carbon source.

\section{Lignin}

Substances used

The lignin preparations used were extracted from samples of brown rotted spruce wood (Picea excelsa) with a lignin content of about $80 \%$ according to sulphuric acid lignin determinations. Two per cent $\mathrm{NaOH}$ in ethanol at room temperature $\left(20^{\circ}\right)$ was used for extraction in accordance with the method of Phillips (1928) as described by Brauns (1952). The crude lignin fraction, precipitated with hydrochloric acid, represented about $8 \%(\mathrm{w} / \mathrm{w})$ of the brown rotted wood on a dry matter basis. After washing until a negative reaction was attained for $\mathrm{Cl}$ ion in the wash water, and drying, the lignin precipitate was dissolved in purified dioxan by stirring for $4 \mathrm{hr}$, to give a solution of about $1 \%(\mathrm{w} / \mathrm{v})$. Undissolved particles were removed by centrifugation and the clear supernatant fluid evaporated to small volume in a rotating evaporator under reduced pressure. The lignin was precipitated by dropping the concentrated solution into $10 \mathrm{vol}$. anhydrous ether, accompanied by vigorous stirring for $30 \mathrm{~min}$. The precipitate was collected in the centrifuge, washed twice with anhydrous ether, once with light petroleum $\left(100-120^{\circ}\right)$ and finally once with light petroleum $\left(40-60^{\circ}\right)$. The final product, after drying to constant weight over

Table 1. Analytical data on the lignin preparations isolated from brown rotted spruce wood (Picea excelsa)

The figures are percentages on a dry matter basis.

\begin{tabular}{|c|c|c|}
\hline Preparation & $\mathbf{A L D}_{p}$ & $\mathbf{A L H}_{y}$ \\
\hline $\begin{array}{l}\text { Lignin ( } 72 \% \text { sulphuric acid method as } \\
\text { described by Hägglund (1951)) }\end{array}$ & $98 \cdot 8$ & $96 \cdot 0$ \\
\hline $\begin{array}{l}\text { Methoxyl (method of Vieböck \& } \\
\text { Schwappach as described by Houben } \\
\text { \& Weyl, 1953) }\end{array}$ & $13 \cdot 80$ & $13 \cdot 57$ \\
\hline $\begin{array}{l}\text { Carbohydrate (determined as glucose } \\
\text { with anthron method according to } \\
\text { Trewellyan \& Harrison, 1952) }\end{array}$ & 0.50 & 0.49 \\
\hline
\end{tabular}


sulphuric acid and paraffin, was a light brown powder and represented about $2.5 \%$ of the dry wt. of the brown rotted wood specimen. Analytical data of the preparation specimens $\mathrm{ALD}_{p}$ and $\mathrm{ALH}_{p}$ from two samples of brown rotted spruce wood, are given in Table 1.

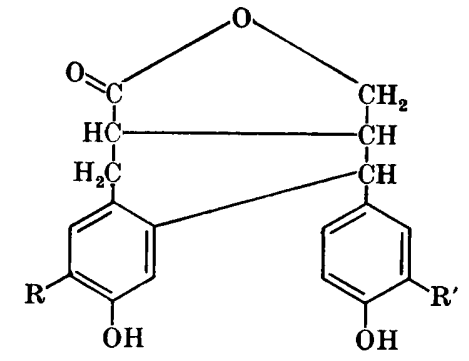

I. $\mathrm{R}=\mathrm{R}^{\prime}=\mathrm{OCH}_{3}$ : $\alpha$-conidendrin.

II. $\quad \mathrm{R}=\mathrm{R}^{\prime}=\mathrm{OH}: \alpha$-conidendrol (trans-lactone).

III. $\mathbf{R}=\mathrm{R}^{\prime}=\mathrm{OH}: \beta$-conidendrol (cis-lactone).

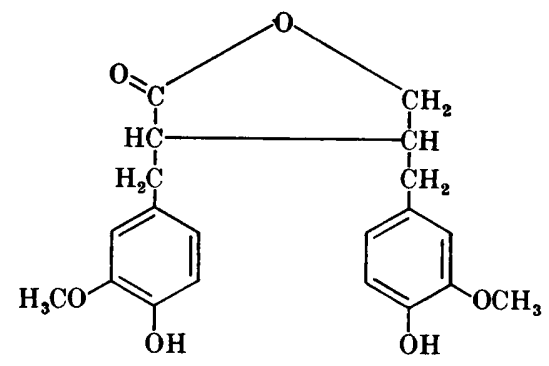

IV. Matairesinol.<smiles>[R]c1cc(C(O)CO)ccc1O</smiles>

V. $R=H, R^{\prime}=\mathrm{OH}$ : isotaxiresinol.

VI. $\mathrm{R}=\mathrm{OH}, \mathrm{R}^{\prime}=\mathrm{OCH}_{3}$ : iso-olivil.

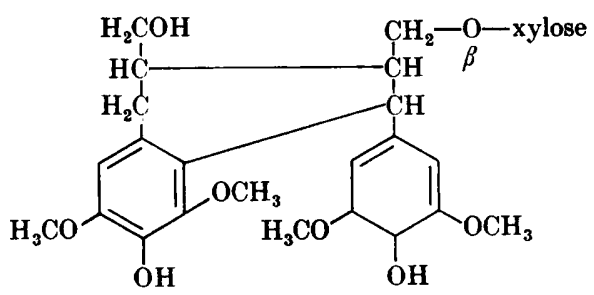

VIII. Lignan xyloside.<smiles>COc1cc(OCC(O)C(O)c2ccc(O)c(OC)c2)ccc1O</smiles>

VII. Olivil.

Fig. 1. Structure of investigated lignans and lignan derivatives.

Lignans and lignan derivatives. (The roman numerals refer to the structures presented in Fig. 1.)

$\alpha$-Conidendrin (I) was supplied by the generosity of the Crown and Zellerbach

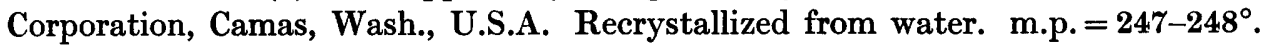
$\alpha$-Conidendrol (II) (trans-configuration at the lactone group), purum quality, Fluka AG, Switzerland. m.p. $=157-161^{\circ}$.

$\beta$-Conidendrol (III) (cis-configuration at the lactone group), purum quality, Fluka AG, Switzerland. m.p. $=244-248^{\circ}$. 
Matairesinol (IV), as well as compounds V-VIII, was a generous gift from Professor H. Erdtman, Stockholm. m.p. $=113-114^{\circ}$.

Isotaxiresinol $(\mathrm{V})$, m.p. $=165-168^{\circ}$.

Iso-olivil (VI), m.p. $=154-157^{\circ}$.

Olivil (VII), recrystallized from acetone, m.p. 143-145 .

Lignan xyloside (VIII), isolated from the sapwood of Sorbus aucuparia (Arya, Erdtman, Krolikowska \& Norin, 1962), m.p. $=159-161^{\circ}$.

Guaiacyl- $\beta$-coniferyl ether (IX). A small sample synthesized by Dr H. Ishikawa (Department of Organic Chemistry and Enzymology, Fordham University, New York) was kindly supplied by Professor F. F. Nord.

Monomeric compounds. (The roman numerals refer to the structures presented in Fig. 2.)<smiles>COc1cc(CC(=O)O)ccc1O</smiles>

X. Guaiacylpyruvic acid.<smiles>[R]c1ccc(C=O)cc1[R]</smiles>

XV. $\mathbf{R}=\mathrm{OCH}_{3}, \mathrm{R}^{\prime}=\mathrm{OH}$ : isovanillin.

XVI. $\mathbf{R}=\mathrm{OH}, \mathrm{R}^{\prime}=\mathrm{OCH}_{3}$ : vanillin.

XVII. $\quad \mathbf{R}=\mathbf{R}^{\prime}=\mathrm{OCH}_{3}$ : veratryl aldehyde.<smiles>COc1cc(CCC(=O)O)ccc1O</smiles>

XXII. Hydrocaffeic acid.<smiles>[R]c1cc(OC)c(O)c(OC)c1</smiles>

XXIV. $R=\mathrm{COOH}$ : syringic acid.

$\mathrm{XXV} . \quad \mathrm{R}=\mathrm{CHO}$ : syringyl aldehyde.

XXVI. $\mathrm{R}=\mathrm{CH}=\mathrm{CH}-\mathrm{COOH}$ : sinapic acid.<smiles>[R]c1ccc(C(=O)O)cc1[R]</smiles>

XI. $\mathrm{R}=\mathrm{OCH}_{3}, \mathrm{R}^{\prime}=\mathrm{OH}$ : isovanillic acid.

XII. $R=O H, R^{\prime}=\mathrm{OCH}_{3}$ : vanillic acid.

XIII. $\mathrm{R}=\mathrm{OCH}_{3}, \mathrm{R}^{\prime}=\mathrm{H}$ : anisic acid.

XIV. $\mathrm{R}=\mathrm{R}^{\prime}=\mathrm{OCH}_{3}$ : veratric acid.<smiles>[R]c1ccc(/C=C/C(=O)O)cc1[R]</smiles>

XVIII. $\quad R=R^{\prime}=\mathrm{OH}$ : caffeic acid.

XIX. $\quad R=O H, R^{\prime}=\mathrm{OCH}_{3}$ : ferulio acid.

XX. $\mathrm{R}=\mathrm{OCH}_{3}, \mathrm{R}^{\prime}=\mathrm{OH}$ : isoferulic acid.

XXI. $R=R^{\prime}=O_{C H}$ : 3-4-dimethoxycinnamic acid<smiles>COc1cc(/C=C/CO)ccc1O</smiles>

XXIIr. Coniferyl alcohol.<smiles>COc1cc(CO)ccc1O</smiles>

XXVII. Vanillyl aloohol.

Fig. 2. Structure of investigated monomeric compounds. 
Guaiacylpyruvic acid $(\mathbf{X})$, same source as compound IX.

Isovanillic acid (XI). A preparation was supplied by Professor T. Enkvist, Helsinki. After boiling with active carbon, the isovanillic acid was recrystallized from water. White needles, m.p. $=247-248^{\circ}$.

Substances XII-XXVII listed below were commercial preparations from Fluka AG, Switzerland (except vanillin from British Drug Houses Ltd.) the grades of purity being listed: vanillic acid (XII), purum; anisic acid (XIII), purum; veratric acid (XIV), puriss.; isovanillin (XV), purum; vanillin (XVI), p.a.; veratryl aldehyde (XVII), purum; caffeic acid (XVIII), purum; ferulic acid (XIX), purum; isoferulic acid (XX), puriss.; 3-4-dimethoxy-cinnamic acid (XXI), puriss.; hydrocaffeic acid (XXII), purum; coniferyl alcohol (XXIII), purum; syringic acid (XXIV), purum; syringyl aldehyde (XXV), purum; sinapic acid (XXVI), puriss.; vanillyl alcohol (XXVII), pract., recrystallized, m.p. $=113 \cdot 5-115^{\circ}$.

\section{RESULTS}

\section{Oxidation of $\alpha$-conidendrin}

The enzyme system for oxidation of $\alpha$-conidendrin was adaptive in organisms of strains Ks1741 and Ms 144412, but present in strain 0811 independently of whether $\alpha$-conidendrin was present during growth or not. This is illustrated in Fig. 3 and Fig. 4, respectively. Figure 3 records the oxygen uptake with $\alpha$-conidendrin as substrate for organisms of strain Ms 144412, produced on yeast extract, and yeast extract $+\alpha$-conidendrin. The figure shows that organisms grown on yeast extract did not oxidize $\alpha$-conidendrin until after a lag of $3 \mathrm{hr}$, when oxygen uptake started at a rate comparable to that noted for organisms grown on $\alpha$-conidendrin. The adaptability to $\alpha$-conidendrin oxidation was preserved in the cultures of $\mathrm{Ks} 1741$ and MS144412 during more than 20 monthly transfers into semi-solid KYE-agar (Sundman, 1962) without $\alpha$-conidendrin.

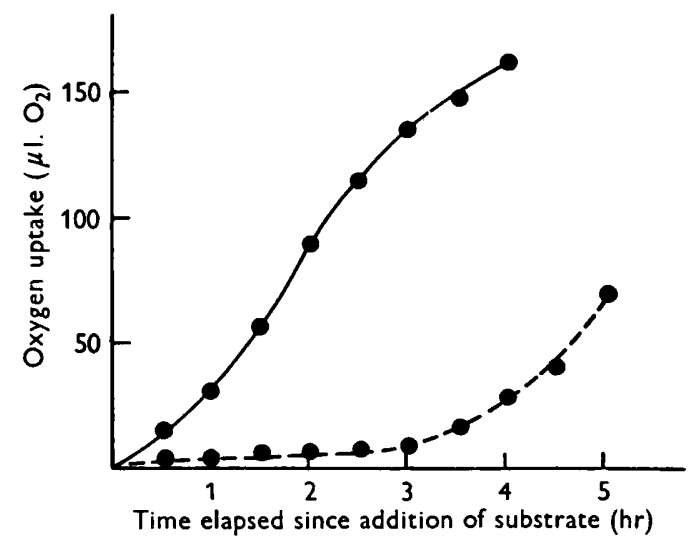

Fig. 3. Oxygen uptake by washed organisms of strain Ms 144412 with $\alpha$-conidendrin as substrate, showing effect of adaptation to the substrate during growth. Endogenous oxygen uptake was subtracted.

Number of viable organisms added to each flask:,$- 2.5 \times 10^{10} ;-\ldots-, 3.6 \times 10^{10}$.

Amount of substrate: $1 \mathrm{mg} .=2 \cdot 8 \mu \mathrm{mole}$. - Organisms produced on a $\alpha$-conidendrin-containing medium; ---, organisms produced without $\alpha$-conidendrin. 
The type of $\alpha$-conidendrin oxidation found in strain 0811 is illustrated in Fig. 4. It is seen that oxygen uptake with $\alpha$-conidendrin as substrate starts immediately with both yeast extract-grown organisms and organisms adapted to $\alpha$-conidendrin during growth. This constitutive ability of lignan oxidation was not found to be a stable characteristic of strain o811; a stock culture kept on semi-solid KYE agar proved after 7 monthly subcultures on this $\alpha$-conidendrin-free medium to be unable to utilize $\alpha$-conidendrin, in growth experiments, or to take up oxygen with $\alpha$ conidendrin as substrate in Warburg experiments. The ability of $\alpha$-conidendrin oxidation was irreversibly lost by this culture, and could not be restored by growing
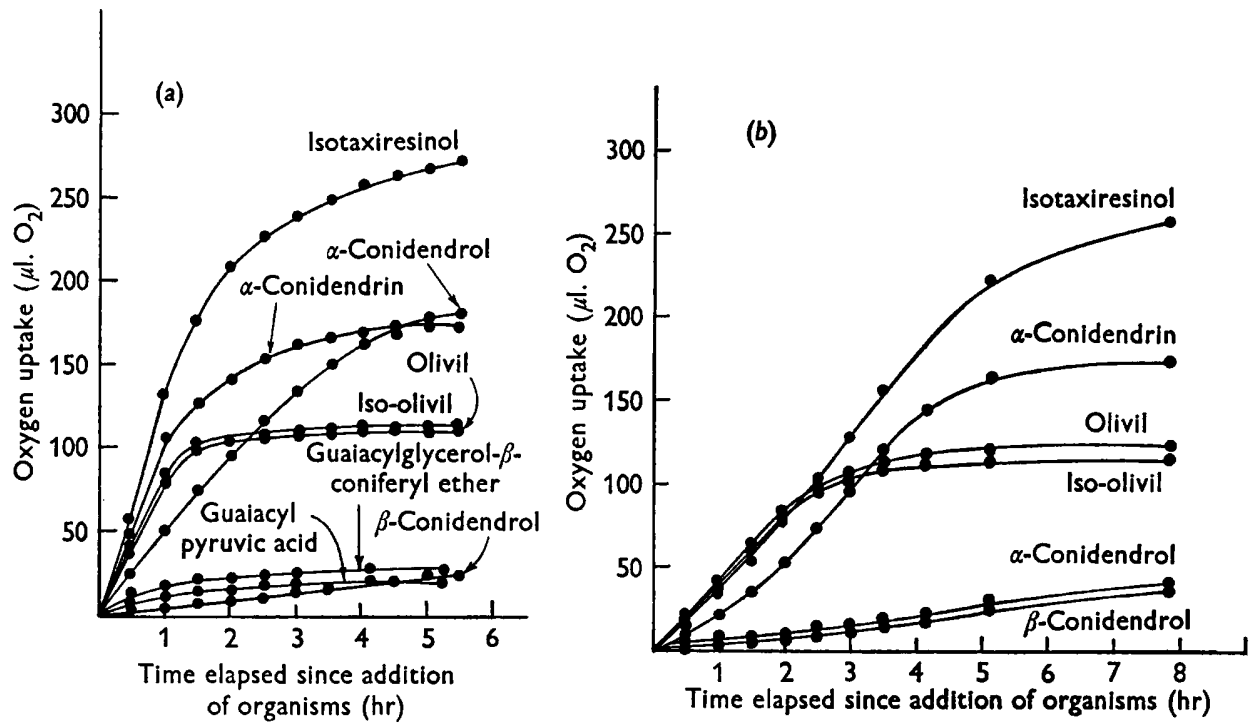

Fig. $4 a, b$. Oxygen uptake by washed organisms of strain 0811 , with various lignans, guaiacylglycerol- $\beta$-coniferyl ether, and guaiacylpyruvic acid, showing the lignanolytic capacity of this strain to be present whether the organisms were produced on $\alpha$-conidendrin or not. Endogenous oxygen uptake was subtracted. Amount of substrate: $1 \mu$ mole. $a$, Organisms produced on $\alpha$-conidendrin-containing medium. Number of viable organisms added to each flask: $2.7 \times 10^{10} . b$, Organisms produced without $\alpha$-conidendrin. Number of viable organisms added to each flask: $3.5 \times 10^{10}$.

it for several passages on a conidendrin-containing medium, or by shaking washed organisms of the culture with $\alpha$-conidendrin crystals for several hours. In another stock culture of the strain $0811 \mathrm{kept}$ on $\alpha$-conidendrin as sole carbon + energy source the ability to oxidize $\alpha$-conidendrin had been preserved. This culture has since during several passages on conidendrin-free medium preserved the constitutive type of $\alpha$-conidendrin oxidation illustrated in Fig. 4.

Previously reported results indicate that the adaptive and constitutive attack on $\alpha$-conidendrin proceed along similar metabolic paths; paper chromatographic analysis of acid ether extracts of $\alpha$-conidendrin cultures of the constitutively lignanolytic strain 0811 and of the adaptive strains $\mathrm{ks1741}$, respectively, has shown identical patterns of phenolic degradation products (Sundman, 1962).

The oxygen uptake with $\alpha$-conidendrin is unaffected by $\mathrm{pH}$ variations in the 
range $\mathrm{pH} 4 \cdot 6-7 \cdot 6$. In repeated experiments, total oxygen uptakes varying between 13 and 17 atoms of oxygen/molecule $\alpha$-conidendrin have been noted. This corresponds to $36-39 \%$ of the oxygen required for complete oxidation to $\mathrm{CO}_{2}$ and $\mathrm{H}_{2} \mathrm{O}$.

\section{Oxidation of other lignans}

All the bacteria examined utilized in addition to $\alpha$-conidendrin the lignans isotaxiresinol (V), iso-olivil (VI) and olivil (VII), whereas matairesinol (IV) and the lignan xyloside (VIII) remained unutilizable. With the adaptive strains Ks 1741 and Ms 144412, adaptation to $\alpha$-conidendrin resulted in simultaneous adaptation to the

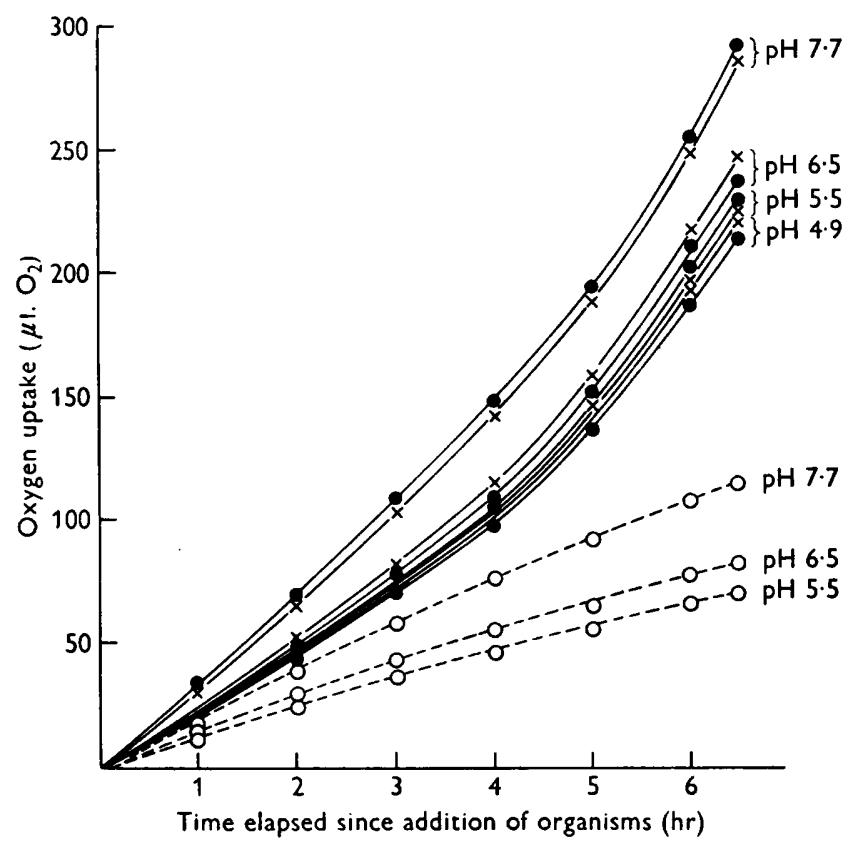

Fig. 5. Oxygen uptake by washed organisms of strain ks 1741 adapted to $\alpha$ conidendrin during growth, with olivil and iso-olivil at various $\mathrm{pH}$ values, showing independence of $\mathrm{pH}$ in the range $\mathrm{pH} 4 \cdot 9-7 \cdot 7$, and identity of olivil and iso-olivil oxidation. $\bigcirc \cdots-.-0$, Endogenous; $x-\times$, olivil; --0 , iso-olivil.

compounds isotaxiresimol, iso-olivil, and olivil. Organisms not adapted to $\alpha$ conidendrin during growth started oxidation of these lignans after a longer time lag than that required for oxidation of $\alpha$-conidendrin to start. Strain o811 required no adaptation for oxidation of the lignans (Fig. 4). The above-mentioned culture of strain 0811 which had lost the ability of $\alpha$-conidendrin oxidation had simultaneously lost the ability to oxidize the other lignans.

Matairesinol (IV), which is not oxidized by the present bacteria, differs structurally from $\alpha$-conidendrin only in the lack of the 6- $\alpha$ carbon-carbon bond (see Fig. 1). Presence of this linkage between the two guaiacylpropane units of the lignan molecule thus seems to be a requirement for the lignanolytic enzyme systems of the bacteria investigated here. It was assumed (Sundman, 1964) that the 6- $\alpha$ bond is preserved in the isovanillic acid formed in the present type of lignan decomposition. Of the lignans other than $\alpha$-conidendrin, which are oxidized, iso-olivil and iso- 
taxiresinol like conidendrin contain the 6- $\alpha$ bond. The variation in side chain structure (see Fig. 1) between $\alpha$-conidendrin on one side and isotaxiresinol and isoolivil on the other, does not influence the oxidation of the compounds as far as rate of oxygen uptake is concerned (Fig. 4).

Special interest was paid to the oxidation of olivil in which the 6- $\alpha$ 'isolignan bond' is not present. Repeated measurements of oxygen uptake with olivil and iso-olivil as substrates showed that the compounds are oxidized identically. Examples of this are seen in Fig. 5. The oxygen uptakes with olivil and iso-olivil as substrates at various $\mathrm{pH}$ values are presented in the figure without subtraction of the corresponding endogenous values in order to demonstrate the identity of olivil
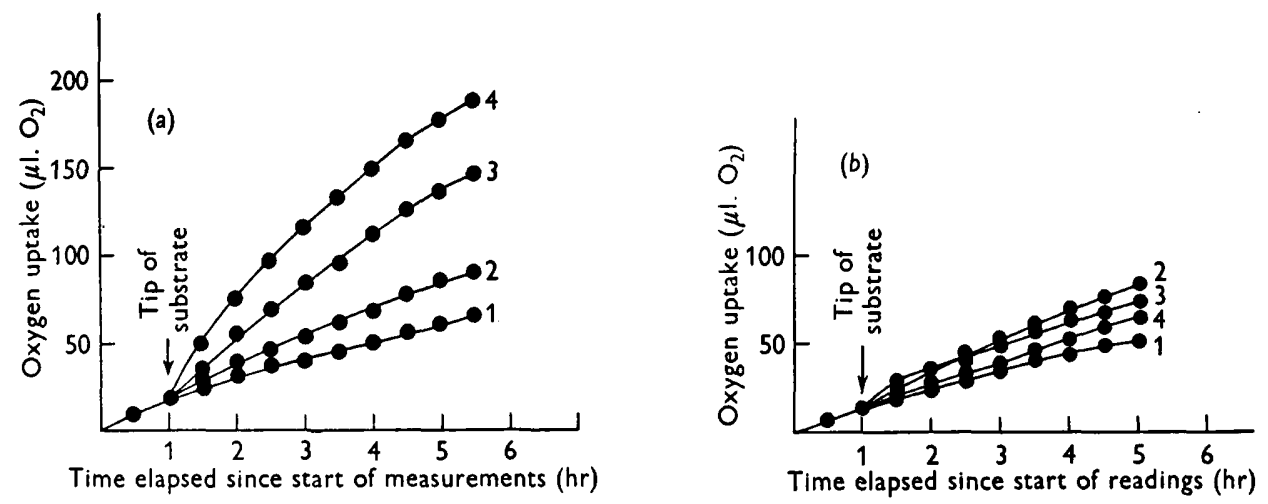

Fig. $6 a, b$. Oxygen uptake by washed organisms of strain Ks 1741, with $\alpha$ conidendrin, $\alpha$ - or $\beta$-conidendrol as substrate, showing simultaneous adaptation to $\alpha$-conidendrol, and $\alpha$-conidendrin.

Amount of substrate: $1 \mu$ mole. $a$, Organisms produced on $\alpha$-conidendrin-containing medium. Number of viable organisms added to each flask: $6 \cdot 15 \times 10^{10}$. Storage of organisms prior to measurement: curves 1-3, 1 day; curve 4, 7 days. $b$, Organisms produced without $\alpha$-conidendrin. Number of viable organisms added to each flask: $6.45 \times 10^{10}$. Organisms stored 3 days prior to measurement. 1, Endogenous respiration; $2, \beta$-conidendrol; $3, \alpha$-conidendrol; $4, \alpha$-conidendrin.

and iso-olivil oxidation. If the increase of endogenous respiration with increasing $\mathrm{pH}$ is taken into account, no influence of the $\mathrm{pH}$ variation between 4.9 and $7 \cdot 7$ can be established. This is in full conformity with the results obtained with $\alpha$-conidendrin.

Olivil and iso-olivil contain different types of carbon skeleton, and it has been thought that they represent two biogenetic categories of lignans. The structural difference is reflected, for instance, in the varying yields of vanillin obtained upon nitrobenzene oxidation: $86 \%(\mathrm{w} / \mathrm{w})$ from olivil, and $3 \%(\mathrm{w} / \mathrm{w})$ from iso-olivil (Leopold \& Malmström, 1951). Olivil is readily subject to isomerization when boiled with dilute acids, and iso-olivil is then obtained (Erdtman, 1955). The oxygen uptake, evidently identical, with olivil and iso-olivil observed for the present lignanolytic bacteria, can be explained by assuming that the bacteria bring about the isomerization olivil $\rightleftarrows$ iso-olivil. Should this explanation prove to be valid, a closer biological relationship exists between the formally different lignan groups than a purely chemical approach might predict. Continued work on the mode of action upon olivil and iso-olivil by the present bacteria is in progress. 
The oxidation of the conidendrols is exemplified in Fig. 6. The slight oxygen uptake with $\beta$-conidendrol, similar for $\alpha$-conidendrin-adapted organisms (Fig. 6a) and for organisms not adapted to $\alpha$-conidendrin (Fig. $6 b$ ) was observed with all the strains studied. The increased oxygen uptake by $\alpha$-conidendrin-active organisms in the presence of $\alpha$-conidendrol (Fig. $6 a$ ) was occasionally observed in experiments with the strains Ks1741 and Ms144412, but found to be a constant feature of strain o811, if grown on medium containing $\alpha$-conidendrin. It should be stressed that whereas the ability to oxidize the methoxyl-containing lignans $\alpha$-conidendrin, olivil, iso-olivil and isotaxiresinol is a constitutive feature of strain o811, the increased oxygen uptake with $\alpha$-conidendrol requires adaptation to $\alpha$-conidendrin during growth. The organisms of the $\alpha$-conidendrin-active 0811 culture, produced on yeast extract and glucose, oxidize the lignans without lag, but are incapable of bringing about the increased oxygen uptake with $\alpha$-conidendrol (Fig. $4 b$ ). Conversely, organisms of this strain produced on $\alpha$-conidendrin oxidize $\alpha$-conidendrol without lag at a rate comparable with that of lignan oxidation (Fig. 4a). It was therefore concluded that $\alpha$-conidendrin decomposition by strain 0811 might, as an alternative to the path over isovanillic acid, be initiated by metabolic steps which substitute the methoxyl groups by hydroxyls thus resulting in $\alpha$-conidendrol formation.

The lignan moiety of lignan xyloside (VIII) has a structure which contains the isolignan 6- $\alpha$ bond and a side chain configuration like isotaxiresinol, but the aromatic rings have a syringyl structure with additional methoxyl groups in the 5 positions. The substance was included in the study with a view to determining whether the lignanolytic activity of the present bacteria is restricted to guaiacylpropane type (soft wood) lignans, or whether syringylpropane (hard wood) lignans are also oxidized. As previously mentioned none of the bacteria was able to oxidize the lignan xyloside. It was found that the bacteria oxidize xylose in respirometric experiments, and proliferate on xylose as the sole energy source. Since the lignan xyloside is not oxidized, it can be concluded that the bacteria do not split the molecule into xylose and lignan. This is in close accord with the inability of the present organisms to proliferate on sucrose, or lactose, although glucose and galactose serve as energy sources (Sundman, 1964), indicating a lack of $\beta$-carbohydrases in the studied Agrobacteria.

Guaiacylglycerol- $\beta$-coniferyl ether (IX), as Ishikawa, Schubert \& Nord $(1963 a, b)$ and Fukuzumi (1960) have demonstrated, is released from lignin, and further metabolized by white-rot fungi. A trial was made with the compound as substrate for organisms of strains o811, actively oxidizing the lignans (Fig. 4a). Only a slow oxygen uptake was established.

\section{Oxidation of the lignin preparations}

Table 2 gives a summary of the results obtained with lignin preparations $\mathrm{ALD}_{p}$ and $\mathrm{ALH}_{p}$. As the table illustrates, the preparations were slowly oxidized by strains Ks1741 and o811, $\mathrm{ALD}_{p}$ more easily than $\mathrm{ALH}_{p}$. The oxygen uptake by MS144412 organisms was increased too slightly to allow of positive conclusions. A response to the presence of the lignin preparation during growth was recorded for strain Ks1741 as an increased oxygen uptake with both lignin preparations for organisms harvested from the lignin-containing substrate, taken against the oxygen 
uptake by organisms produced on yeast extract and glucose. The results for strain 0811 were contradictory as far as adaptive response to the presence of lignin in the medium was concerned. The parallel to adaptive contra constitutive degradation of lignans in strains $\mathrm{ks} 1741$ and 0811 is of interest.

Table 2. Results of respirometric experiments with $1 \mathrm{mg}$. of lignin preparations $A L D_{p}$ and $A L H_{p}$ as substrates for washed, lignin-adapted, and non-adapted organisms

\begin{tabular}{|c|c|c|c|c|c|}
\hline \multirow[b]{3}{*}{ Strain } & \multirow{3}{*}{$\begin{array}{l}\text { Lignin } \\
\text { present } \\
\text { in growth } \\
\text { medium* }\end{array}$} & \multirow{3}{*}{$\begin{array}{l}\text { Number of } \\
\text { organisms } \\
\times 10^{-10}\end{array}$} & \multicolumn{3}{|c|}{ Oxygen uptake } \\
\hline & & & \multicolumn{2}{|c|}{$\begin{array}{l}Q_{\mathrm{O}_{2}}\left(10^{10} \text { organisms }\right) \text {. Figures in } \\
\text { brackets indicate } \mu \mathrm{l} . \mathrm{O}_{2} \text {-uptake } \\
\text { in } 3.5 \mathrm{hr} \dagger\end{array}$} & \multirow{2}{*}{$\begin{array}{c}\text { Endogenous } \\
\mu \mathrm{l} . \mathrm{O}_{2} \text { in } \\
3.5 \mathrm{hr}\end{array}$} \\
\hline & & & $\mathbf{A L D}_{p}$ & $\mathbf{A L H}_{p}$ & \\
\hline \multirow[t]{2}{*}{ Ks 1741} & - & $4 \cdot 8$ & $1 \cdot 31(22)$ & $0.71(12)$ & 39 \\
\hline & + & $2 \cdot 2$ & $4.03(31)$ & $2 \cdot 73(21)$ & $\mathbf{3 6}$ \\
\hline \multirow[t]{2}{*}{ o 811} & - & $3 \cdot 5$ & $4.07(50)$ & $0 \cdot 41(5)$ & 43 \\
\hline & + & $1 \cdot 9$ & $3.78(25)$ & $1.51(10)$ & 32 \\
\hline \multirow[t]{2}{*}{ MS 144412} & - & 0.9 & $1 \cdot 27 \quad(4)$ & $0 \cdot 32(1)$ & 32 \\
\hline & + & $1 \cdot 0$ & 2.58 & $1 \cdot 43(5)$ & 27 \\
\hline
\end{tabular}

* The lignin-free medium KYE-agar contained $0.2 \%(w / v)$ Difco yeast extract, and $0.2 \%$ $(w / v)$ glucose (Sundman, 1964). The lignin-containing medium contained in addition $0.05 \%$ $(\mathrm{w} / \mathrm{v})$ of lignin preparations $\mathrm{ALD}_{p}$ and $\mathrm{ALH}_{p}$.

$\dagger$ Endogenous respiration as in last column and autoxidation of lignin, $3 \mu \mathrm{l}$. for $\mathrm{ALD}_{p}$ and $4 \mu \mathrm{l}$. for $\mathrm{ALH}_{p}$, were subtracted.

\section{Oxidation of phenolic monomers}

The compounds X-XXVII were included in the investigation partly with a view to finding possible intermediates in the breakdown of $\alpha$-conidendrin, and partly to acquire knowledge of the oxidative properties of the lignanolytic Agrobacteria. The oxygen uptake with a number of non-methoxylated phenolic compounds has been previously reported (Sundman, 1964).

As regards the possibility that the monomers are intermediates in the metabolic sequences of $\alpha$-conidendrin decomposition, it should be pointed out that adaptation to conidendrin caused no simultaneous adaptation to any of the monomers, except to isovanillin and to isovanillic acid (Fig. $7 a, b$ ).

Of the aldehydes investigated vanillin, syringylaldehyde, veratrylaldehyde were oxidized in a similar manner by strains ks1741 and o811. The oxygen uptake was completed within $60 \mathrm{~min}$., and corresponded to slightly more than one atom of oxygen per molecule of aldehyde. Isovanillin was oxidized in the same way by organisms of strain Ks1741 if not adapted to $\alpha$-conidendrin during growth. This type of aldehyde oxidation is illustrated in Fig. $7 a$ (curve no. 2) and Fig. $7 b$ (curves nos. 2 and 5). Similar oxidation of the aldehydes, in spite of differently substituted ring structure, pointed to a common type oxidation restricted to the aldehyde group, which was assumed to be oxidized to carboxyl. When a slight oxygen uptake observed with the corresponding carboxyl compounds was subtracted from the oxygen uptake noted for the aldehydes, on the assumption that the aldehydes are oxidized to corresponding acids, a total oxygen consumption for the reaction aldehyde $\rightarrow$ acid was obtained, as indicated in Table 3 . The figures in the table 
indicate that the oxygen uptake observed for strain ks 1741 was markedly less than the theoretical amount: 1 atom of oxygen for every molecule of aldehyde.

For study of the reaction products of aldehyde oxidation by strain Ks 1741, the contents of Warburg flasks were analysed as follows: when manometer readings indicated that the oxygen uptake with aldehyde as substrate had diminished to the same rate as endogenous respiration, the content was pipetted off and centrifuged. The supernatant was evaporated to small volume, and the concentrated supernatant quantitatively applied as two spots on different sheets of paper previously buffered to $\mathrm{pH} 6.5$ with $\mathrm{M} / 15$ phosphate buffer. Pure aldehydes, the corresponding acids and vanillyl alcohol were applied as standards. The chromatograms were developed by ascending technique with water-saturated $n$-butanol.
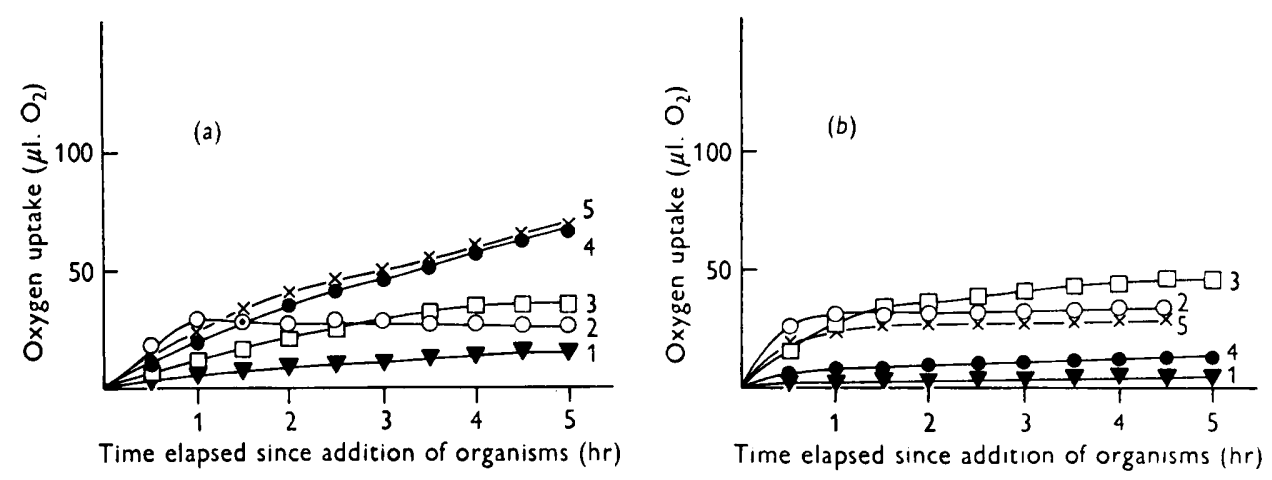

Fig. 7a, $b$. Oxygen uptake by washed, organisms of strain ks 1741, with vanillin acid, vanillin, coniferylalcohol, isovanillic acid, or isovanillin as substrate showing adaptation to isovanillin and isovanillic acid upon adaptation to $\alpha$-conidendrin during growth. Endogenous oxygen uptake was subtracted.

Amount of substrate: $2 \mu$ mole. $a$, Organisms produced on $\alpha$-conidendrin-containing medium. Number of viable organisms added to each flask: $2.4 \times 10^{10}$. Storage prior to measurement: curve 1, 4 days; curve 2, 9 days; curve 3, 11 days; curve 4, 4 days ; curve 5, 9 days. $b$, Organisms produced without $\alpha$-conidendrin. Number of viable organisms added to each flask : $4.7 \times 10^{10}$. Storage prior to measurement: curves 1,3 , and 4, 1 day; curves 2 and 5,3 days. $1, \nabla$, Vanillic acid; 2,0 , vanillin; $3, \square$, coniferylalcohol; 4 , $\bigcirc$, isovanillic acid; $5, \times$, isovanillin.

Table 3. Oxygen uptake evaluated as atoms of oxygen per molecule of aldehyde for reaction $\mathrm{RCHO} \rightarrow \mathrm{RCOOH}$, as calculated from the difference between total oxygen uptakes observed for $\mathrm{RCHO}$ and $\mathrm{RCOOH}$

\begin{tabular}{|c|c|c|}
\hline \multirow{2}{*}{$\boldsymbol{R}$} & \multicolumn{2}{|c|}{$\begin{array}{c}\text { Oxygen uptake, atoms of oxygen per } \\
\text { molecule of aldehyde }\end{array}$} \\
\hline & Strain o 811 & Strain ks 1741 \\
\hline Vanillyl- & $1 \cdot 04$ & $0 \cdot 64$ \\
\hline Isovanillyl- & - & $0 \cdot 87$ \\
\hline Syringyl- & 0.81 & 0.79 \\
\hline Veratryl- & 0.89 & 0.67 \\
\hline
\end{tabular}

One of the runs was sprayed with 2-4-dinitrophenyl-hydrazine (carbonyl reagent) and the other with diazotized sulphanilic acid (phenol reagent). For the veratryl aldehyde run, rhodamin $B$ (reagent for acids) was used for spraying the second run.

It was found that no detectable aldehyde or other carbonyl compound remained 
in the samples. Instead, there were strong spots coinciding with the corresponding acids: vanillic, isovanillic, and syringic acid appeared on the chromatograms after spraying with diazotized sulphanilic acid. In addition, a second spot of higher $\boldsymbol{R}_{\boldsymbol{f}}$ value, detectable with diazotized sulphanilic acid, was present on the runs. In the vanillin run, this spot $\boldsymbol{R}_{f}=\mathbf{0} \cdot 84$ was identical with the spot of vanillyl alcohol. From isovanillin, there had been formed a substance with $\boldsymbol{R}_{f}=\mathbf{0 . 8 3}$, and from syringyl aldehyde a substance at $R_{f}=0.75$, through the activity of the bacteria. These two compounds have not yet been identified, owing to the lack of standards available for comparison, but there is reason to believe that they might be the corresponding alcohols.

It appears that an intramolecular dismutation of vanillin to vanillyl alcohol and vanillic acid proceeds simultaneously with oxidation of the aldehyde to acid during oxygen uptake. Reactions of this type, leading to the accumulation of vanillyl alcohol, obviously account for the deviation of oxygen uptake from the theoretical amount: 1 atom of oxygen for every molecule of aldehyde, as indicated in Table 3.

The oxidation of aldehyde to acid as exemplified in Fig. $7 a$ for vanillin and in Fig. $7 b$ for vanillin and isovanillin does not provide energy to allow the bacteria to proliferate on the aldehydes as the sole energy source. Neither are bacteria which bring about the negligible oxidation of vanillic acid (Fig. $7 a, b$ ) or the comparable oxidation of isovanillic acid (Fig. $7 b$ ) able to grow on these acids as sole carbon sources. Against this, isovanillic acid can serve as the sole energy + carbon source for organisms adapted to $\alpha$-conidendrin, hence bringing about the increased-type oxidation of isovanillic acid in Fig. $7 a$.

Coniferyl alcohol was oxidized at a comparatively slow rate (Fig. $7 a, b$ ), and a total oxygen uptake corresponding to $1 \cdot 5-1.98$ atoms of oxygen/molecule substrate was recorded. In conformity with the amount of oxygen consumed, ferulic acid was by paper chromatography demonstrated to be the reaction product. No other phenolic compounds except traces of remaining coniferyl alcohol could be demonstrated.

Guaiacylpyruvic acid (X) has been demonstrated as intermediate in the degradation of lignin by white-rot fungi (Fukuzumi, 1959, 1960; Ishikawa et al. 1963a, b) and appears to occupy a central position in the fungal metabolism of lignin. The crude preparation of $(\mathbf{X})$ available was used as substrate with actively lignanolytic o811 organisms (Fig. 4a). The slow oxidation does not indicate that guaiacylpyruvic acid plays an important part in the metabolism of lignans by the agrobacteria examined here.

Vanillyl alcohol, anisic acid, caffeic acid, hydrocaffeic acid and syringic acid are oxidized at a negligible rate, comparable with the oxidation of vanillic acid and isovanillic acid in Fig. $7 b$, corresponding to less than one atom of oxygen per molecule of substrate in $5 \mathrm{hr}$. The cinnamic acid derivatives with the $\alpha-\beta$ unsaturated side chain: ferulic acid, isoferulic acid, 3-4-dimethoxycinnamic acid and sinapic acid, were oxidized by strain Ks1741, isoferulic acid to an extent corresponding to $\mathbf{1 . 6}$ atoms of oxygen/mole substrate and the other acids corresponding to 1 atom oxygen/mole substrate. With organisms of strain 0811 there could be demonstrated no oxidation of the cinnamic acid derivatives. 


\section{DISCUSSION}

The results presented in this paper, in contrast to previously reported results on the topic, show that conidendrin-decomposing bacteria might also be capable of degrading other lignin-related structures. The experiments with other lignans indicate that the specificity relates merely to the carbon skeleton. The 'isolignan' structure, either present in the substrate or resulting from the isomerizing ability of the bacteria, seems to be a requirement of the organisms under investigation.

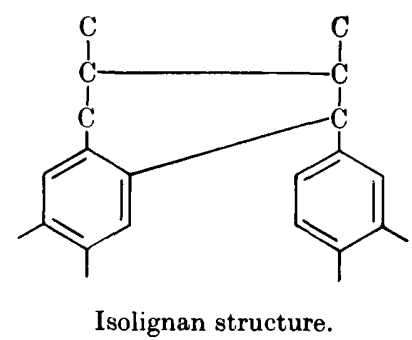

The simultaneous adaptation to the other lignans upon growth on conidendrincontaining medium can be explained either by the assumption that the other lignans are intermediates in the conidendrin-decomposition, or, more likely, in accordance with the ideas of Stanier, Sleeper, Tsuchida \& MacDonald (1950), as a back adaptation over reversible steps to a possible common substrate for the lignanolytic enzyme system of the bacteria.

The identical oxidation of olivil (VII) and iso-olivil (VI) confirmed by paperchromatographic work to be published, indicates that the two isomeric forms are in equilibrium in the presence of the bacteria studied. In work on the constitution of olivil and iso-olivil, Traverso $(1960 \mathrm{a})$ concluded that olivil has the structure of hydroxylariciresinol, i.e. that the ether linkage is situated between side chain carbons $\alpha$ and $\gamma$, and the secondary alcohol group of olivil, accordingly at the side chain carbon $\beta$ as a tertiary OH-group. By way of agreement with this view, it was demonstrated that the structure of iso-olivil contains a tertiary OH-group at side chain $\beta$-carbon (Traverso, 1960b):<smiles>COc1cc(C(C)OC(C)C(O)c2ccc(O)c(OC)c2)ccc1O</smiles>

Olivil.

(Structure according to Traverso, $1960 a$ ).<smiles>COc1cc(Cc2ccc(C(O)CO)cc2OC)ccc1O</smiles>

Iso-olivil.

(Structure according to Traverso, $1960 \mathrm{~b}$ ). 
According to these structures, the isomerization olivil $\rightarrow$ iso-olivil is the same transformation as the isomerization of lariciresinol to isolariciresinol, known to occur under the influence of diluted acids:

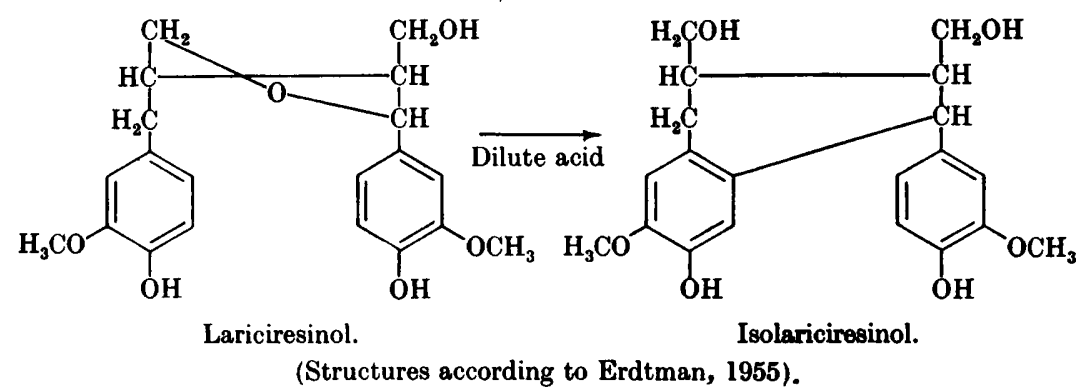

There appear to be possibilities for biological confirmation of the structures proposed by Traverso for olivil and iso-olivil, by means of comparative studies of the activity of the lignanolytic agrobacteria on the pairs olivil-iso-olivil and lariciresinol-isolariciresinol. Such work is in progress.

The results reported in Table 2, concerned with oxidation of the lignin used, indicate that the activity of the lignanolytic bacteria on the modified lignins was rather feeble in comparison with the action on the lignans. Since the biological decomposition of lignin-residue in soil is a slow process, it is possible that bacterial attack of the delayed kind observed in this work could contribute to the biological decomposition of lignin, at least in the modified form in which it occurs in brownrotted wood.

The reduction of aromatic aldehydes under aerobic conditions to alcohols by the present agrobacteria which has been demonstrated to occur when vanillin serves as substrate, and probably working also with isovanillin, syringic aldehyde and veratric aldehyde, has previously been demonstrated with soil fungi which utilize aromatic compounds (Henderson, 1961), with white-rot fungi (Ishikawa et al. $1963 b$ ) and with Aspergillus niger (Raman \& Shanmugasundaram, 1963). The reduction of aldehyde to alcohol, occurring under aerobic conditions simultaneously with the rapid oxidation to acid reported in this paper, forms only a minor part of the aldehyde metabolism of the agrobacteria, under the prevailing conditions. The main part of the aldehyde is transformed to acid.

It appears that the oxidative transformations of aromatic aldehydes, brought about by lignanolytic fungi and bacteria, are the result of reversible processes in which alcohols, aldehydes, and acids are in equilibrium according to the following scheme:

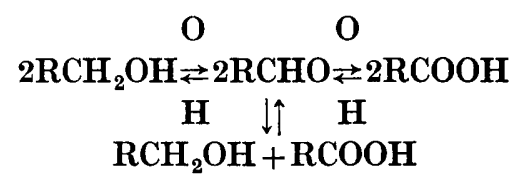

The equilibrium in the reversible processes will favour the more oxidized compound.

The manometric results reported in this paper concerning the oxidation of vanillyl alcohol and vanillin by organisms which are incapable of oxidizing vanillic 
acid indicate that, in comparison with the transformation of aldehyde to acid, the oxidation of alcohol to aldehyde is a slow reaction. The appearance of alcohol at the time when the rapid oxidation of aldehyde to acid has ceased, can be explained by assuming, either that a Cannizzaro-type dismutation of the aldehyde provides the alcohol, or that a small fraction of the aldehyde is reduced to alcohol, this being accumulated in the medium by reason of relatively slow oxidation.

The author is indebted to Professor F. F. Nord and Dr H. Ishikawa for the gift of samples of guaiacylglycerol- $\beta$-coniferyl ether and 4-hydroxy-3-methoxyphenylpyruvic acid. She further wishes to thank Professor H. Erdtman for the samples of lignans. The work was supported by grant No. FG-Fi-108-60 from the U.S. Department of Agriculture. This is the third paper in a series on microbial decomposition of lignins. For previous papers see Sundman $(1962,1964)$.

\section{REFERENCES}

Arya, V. P., Erdtman, H., Krolikowska, M. \& Norin, T. (1962). A lignan xyloside from the sapwood of Sorbus aucuparia L. Acta chem. scand. 16, 518.

Brauns, F. E. (1952). The Chemistry of Lignin, p. 108. New York: Academic Press Inc.

Erdtman, H. (1955). Lignans. In Modern Methods of Plant Analysis, vol. 3. BerlinGöttingen-Heidelberg: Springer-Verlag.

Freudenberg, K. \& Knof, L. (1957). Die Lignane des Fichtenholzes. Chem. Ber. 90, 2857.

Fukuzumi, T. (1959). Phenol oxidases in the wood-rotting fungi. II. Optimum pH with hydroquinone and oxidation of $p$-hydroxy- $m$-methoxyphenylpyruvic acid by enzyme of Poria subacida. J. Japan Wood Res. Soc. 5, 222.

Fukuzumi, T. (1960). Enzymic degradation of lignin. Part I. Paper-chromatographical separation of intermediate degradation products of lignin by the wood-rotting fungus Poria subacida (Peck) Sacc. Bull. agric. chem. Soc. Japan, 24, 728.

HägGlund, E. (1951). Chemistry of Wood, p. 326. New York: Academic Press Inc.

Henderson, M. E. K. (1961). The metabolism of aromatic compounds related to lignin by some Hyphomycetes and yeast-like fungi of soil. J. gen. Microbiol. 26, 155 .

Houben, Th. \& Weyl, J. (1953). Methoden der organischen Chemie, 4th ed., vol. 2. Stuttgart: Georg Thieme Verlag.

Ishikawa, H., Schubert, W. J. \& Nord, F. F. (1963a). Investigations on lignins and lignification. XXVII. The enzymic degradation of soft wood lignin by white-rot fungi. Archs. Biochem. Biophys. 100, 131.

Ishikawa, H., Schubert, W. J. \& Nord, F. F. (1963b). Investigations on lignins and lignification. XXVIII. The degradation by Polyporus versicolor and Fomes fomentarius of aromatic compounds structurally related to soft wood lignin. Archs. Biochem. Biophys. $100,140$.

Konetzka, W. A., Pelczar, M. J. \& Gottlieb, S. (1952). The biological degradation of lignin. III. Bacterial degradation of alpha-conidendrin. J. Bact. 63, 771.

Konetzka, W. A., Woodings, E. T. \& Stove, J. (1957). Microbial dissimilation of methoxylated aromatic compounds. Bact. Proc. p. 135.

Leopold, B. \& Malmström, I.-L. (1951). Constitution of resin phenols and their biogenetic relations. Part XV. Nitrobenzene oxidation of compounds of the lignan type. Acta chem. scand. 5, 936.

Phillips, M. (1928). The chemistry of lignin. II. Fractional extraction of lignin from corn cobs. J. Am. chem. Soc. 50, 1986.

Pratt, Y. T., Konetzka, W. A., Pelczar, M. J., Jr. \& Martin, W. H. (1953). Biological degradation of lignin. V. Polysaccharide synthesis from $\alpha$-conidendrin. Appl. Microbiol. 1,171 . 
Raman, T. S. \& Shanmugasundaram, E. R. B. (1963). Metabolism of some aromatic acids by Aspergillus niger. J. Bact. 84, 1339.

Stanier, R. Y., Sleeper, B. P., Tsuchida, M. \& MacDonald, D. L. (1950). The bacterial oxidation of aromatic compounds. III. The enzymatic oxidation of catechol and protocatechuic acid to $\beta$-ketoadipic acid. J. Bact. 59, 137.

Sundman, V. (1962). Microbial decomposition of lignins. I. Identification of isovanillic acid as a breakdown product in bacterial degradation of $\alpha$-conidendrin. Finska Kemists. Medd. 71, 26.

Sundman, V. (1964). A description of lignanolytic soil bacteria and their ability to oxidize simple aromatic compounds. J. gen. Microbiol. 36, 171.

Tabak, H. H., Chambers, C. W. \& Kabler, P. W. (1959). Bacterial utilization of lignans. J. Bact. 78, 469.

Traverso, G. $(1960 a)$. Natural resinols. III. Constitution of olivil and derivatives. Gazz. chim. ital. 90, 792.

Traverso, G. (1960b). Natural resinols. IV. Constitution of isoolivil. Gazz. chim. ital. 90, 808.

Trewellyan, W. E. \& Harrison, J. S. (1952). Studies on yeast metabolism. Biochem. J. 50, 298. 